\title{
Harmonic and Wave Maps Coupled with Einstein's gravitation
}

\author{
R. Schimming ${ }^{1 *}$ and Ragab M. Gad ${ }^{2,3} \dagger$ \\ 1 Universität Graeifswald, Institut für Mathematik und informatik, \\ D-17487 Greifswald, Germany \\ 2 Mathematics Department, Faculty of Science, King Abdulaziz University, \\ 21589 Jeddah, KSA \\ 3 Mathematics Department, Faculty of Science, Minia University, \\ 61915 El-Minia, Egypt
}

\begin{abstract}
In this paper we discuss the coupled dynamics, following from a suitable Lagrangian, of a harmonic or wave map $\phi$ and Einstein's gravitation described by a metric $g$. The main results concern energy conditions for wave maps, harmonic maps from warped product manifolds, and wave maps from wave-like Lorentzian manifolds.
\end{abstract}

Keywords: Harmonic map; Wave map; Energy conditions.

\section{Introduction}

Scalar fields on a space or space-time manifold $(X, g)$, which satisfy a linear or nonlinear field equation, attract enduring attention; cf, eg., $[1,2,3]$. The Dirichlet Lagrangian or energy density

$$
e=\frac{1}{2}|d \phi|^{2}=\frac{1}{2} g^{a b}\left(\partial_{a} \phi\right)\left(\partial_{b} \phi\right)
$$

of a one-component scalar field $\phi=\phi(x)$ leads to a linear field equation of Laplace or D'Alembert type. Here we denote

$$
g=g_{a b} d x^{a} d x^{b}, \quad\left(g^{a b}\right):=\left(g_{a b}\right)^{-1}, \quad \partial_{a}:=\frac{\partial}{\partial x^{a}} .
$$

\footnotetext{
*E-mail: rschimming@t-online.de

${ }^{\dagger}$ E-mail: ragab2gad@hotmail.com
} 
The Dirichlet Lagrangian $e$ admits a natural generalization to a multicomponent scalar field $\phi=\phi(x)$ if the range of $\phi$ is suitably geometrized, namely if $i m \phi$ lies in a Riemannian manifold $(Y, h)$. That means, $\phi$ becomes a map $X \rightarrow Y, x \mapsto y$ between a source manifold $(X, g)$ and a target manifold $(Y, h)$. The choice of the Lagrangian

$$
e=e[\phi]=e[\phi, g]=\frac{1}{2}|d \phi|^{2},
$$

where now

$$
|d \phi|^{2}:=\operatorname{tr}\left(\phi^{*} h\right) \equiv g^{a b}\left(\partial_{a} \phi^{i}\right)\left(\partial_{b} \phi^{j}\right) h_{i j}\left(\phi^{k}\right),
$$

leads to the generally semi-linear field equation with Laplace-like or D'Alembertlike principal part

$$
\operatorname{tr}(\nabla d \phi)^{i} \equiv g^{a b} \nabla_{a} \partial_{b} \phi^{i}=0 .
$$

Here we denote $h=h_{i j} d y^{i} d y^{j}$ and the special covariant derivative $\nabla$ is built from $g, h, \phi$ as will be explained in Section 2 .

A map $\phi: X \longrightarrow Y$ between properly Riemannian manifolds $(X, g)$, $(Y, h)$ which satisfies $(1.3)$ is called a harmonic map $[4,5]$. A map $\phi: X \longrightarrow$ $Y$ from a Lorentzian manifold $(X, g)$ to a properly Riemannian manifold $(Y, h)$ which satisfies $(1.3)$ is called a wave map $[3,6,7,8,9]$.

In this paper, we take $(Y, h)$ as fixed background and consider $g$ and $\phi$ as dynamical objects. The dynamics shall follow from the Lagrangian

$$
L=\kappa R-e,
$$

where $R$ denotes the scalar curvature of $g$ and $\kappa \neq 0$ is a coupling constant. Variation with respect to $g$ yields, for $\operatorname{dim} X \geq 3$, the Einstein equation in the form

$$
\kappa \text { Ric }=\phi^{*} h
$$

in components

$$
\kappa R_{a b}=\left(\partial_{a} \phi^{i}\right)\left(\partial_{b} \phi^{j}\right) h_{i j} .
$$

Variation with respect to $\phi$ yields (1.3).

A Lorentzian metric $g$ can be interpreted as gravitation. According to the Kaluza-Klein principle, the space-time manifold $(X, g)$ may have a higher dimension.

A positive definite metric $g$ on $X$ can be given a physical interpretation through a Lorentzian metric constructed from it as follows. Consider the product manifold $\tilde{X}:=\Re \times X$ with points $(t, x)$ and equip it with the 
Lorentzian metric $\tilde{g}:=d t^{2}-g$. Extend $\phi: X \longrightarrow Y$ to $\tilde{\phi}: \tilde{X} \longrightarrow Y$ by setting $\tilde{\phi}(t, x):=\phi(x)$, that means $\tilde{\phi}:=\phi \circ p r_{2}$, where $p r_{2}$ is the projection $\tilde{X} \longrightarrow X$ to the second factor. The field equations $(1.5),(1.3)$ for $(\tilde{X}, \tilde{g}), \tilde{\phi}$ reduce to $(1.5),(1.3)$ for $(X, g), \phi$.

Let us sketch our main results.

- The energy-momentum tensor $T$ of a wave map obeys several energy conditions. In particular, if $v$ is a causal (i.e. non-spacelike) vector field then the momentum one-form $I:=T(\cdot, v)$ is causal again.

- The Einstein equation (1.5) implies that the conditions $\operatorname{Ric}(v, v)=0$, $\operatorname{Ric}(\cdot, v)=0, \phi_{*} v=0$ for a vector field $v$ are equivalent to each other. Moreover, then $v$ is a Ricci collineation, i.e. $£_{v} R i c=0$. Some conclusions are drawn from this latter fact.

- A submersive map $\phi: X \rightarrow Y$ between a pure manifold $X$ and a Riemannian manifold $(Y, h)$ can locally be made to a harmonic or wave map by a suitable choice of a metric $g$ on $X$.

- We study the case of warped product $X={ }^{\prime} X \times{ }^{\prime \prime} X, g={ }^{\prime} g \oplus w^{2}{ }^{\prime \prime} g$. Several propositions are proved by means of the argument that the integral of a Laplace expression over a closed manifold ' $X$ vanishes.

- We study radiation conditions for a Lorentzian manifold $(X, g)$. The Einstein equation (1.5) leads from one condition to a stronger condition.

\section{Preliminaries}

We consider dimensions

$$
m:=\operatorname{dim} X \geq 3, \quad n:=\operatorname{dim} Y \geq 1
$$

and adopt the following index convention: indices $a, b, c, \ldots$ label the components of geometric objects on $X$; indices $i, j, k, \ldots$ label the components of geometric objects on $Y$.

Tensor fields on $Y$ become multi-component scalar fields on $X$ by insertion of $y=\phi(x)$, where $x \in X, y \in Y$. For covariant tensor fields on $Y$ there is also the conventional pull-back map $\phi^{*}$. For instance, the metric $h=h_{i j} d y^{i} d y^{j}$ yields $h \circ \phi$ with components $h_{i j}\left(\phi^{k}\right)=h_{i j}\left(\phi^{k}\left(x^{a}\right)\right)$ on $X$ and 
also the pull-back $\phi^{*} h$ on $X$ with components $\left(\partial_{a} \phi^{i}\right)\left(\partial_{b} \phi^{j}\right)\left(h_{i j}\left(\phi^{k}\right)\right.$. The object $\phi^{*} h$ is called first fundamental form of $\phi: X \longrightarrow Y$.

We will occasionally write

$$
\phi^{*} h=h(d \phi, d \phi)
$$

and also use the bilinear symmetric form $h(\cdot, \cdot)$ with tensorial entries.

Some natural covariant derivative $\nabla$ with components $\nabla_{a}$ is built from $g, h$, $\phi$ according to the following rules

1. $\nabla$ applied to tensor fields on $X$ equals the Levi-Civita derivative ${ }^{g} \nabla$ to $g$. For instance,

$$
\nabla_{a} v^{c}:=\partial_{a} v^{c}+{ }^{g} \Gamma_{a b}^{c} v^{b},
$$

where $v=v^{a} \partial_{a}$ is a vector field on $X$ and ${ }^{g} \Gamma_{a b}^{c}$ are the Christoffel symbols to $\left(g_{a b}\right)$.

2. $\nabla$ applied to tensor fields on $Y$ equals some pull-back of the Levi-Civita derivative ${ }^{h} \nabla$ to $h$. For instance,

$$
\nabla_{a} w^{k}:=\left(\partial_{a} \phi^{i}\right)\left(\partial_{i} w^{k}+{ }^{h} \Gamma_{i j}^{k} w^{j}\right),
$$

where $w=w^{i} \partial_{i}$ is a vector field on $Y$ and ${ }^{h} \Gamma_{i j}^{k}$ are the Christoffel symbols to $\left(h_{i j}\right)$. Here $\nabla_{a} w^{k}$ is understood to depend on $y^{l}=\phi^{l}\left(x^{c}\right)$.

3. There are natural product rules for mixed quantities, the components of which carry both indices $a, b, \ldots$ and $i, j, \ldots$ For instance, the differential $d \phi$ of $\phi: X \longrightarrow Y$ with components $\partial_{a} \phi^{i}$ is a mixed tensor field. The covariant derivative $\nabla d \phi$ of $d \phi$ is called second fundamental form of $\phi$. It has the components

$$
\nabla_{a} \partial_{b} \phi^{k}=\partial_{a} \partial_{b} \phi^{k}-{ }^{g} \Gamma_{a b}^{c} \partial_{c} \phi^{k}+{ }^{k} \Gamma_{i j}^{h}\left(\partial_{a} \phi^{i}\right)\left(\partial_{b} \phi^{j}\right)
$$

and the symmetry property

$$
\nabla_{a} \partial_{b} \phi^{k}=\nabla_{b} \partial_{a} \phi^{k}
$$

More on calculus for maps between (pseudo-) Riemannian manifolds can be found in the literature, e.g. $[4,5]$. 


\section{The field equations}

The field theory for $g$ and $\phi$ considered here is based on the Lagrangian

$$
L=\kappa R-e,
$$

which is the sum of the gravitational Lagrangian $\kappa R$ and the matter Lagrangian $e$. Here $R=R[g]$ denotes the scalar curvature of $g, \kappa \neq 0$ is a coupling constant and $e=e[\phi]=e[g, \phi]$ is given by (1.2).

The idea to couple a harmonic map, formerly also called sigma model, with Einstein's gravitation appeared in $[10,11,12]$ and other early papers.

The following is well-known $[7,8,9,10,11,12,13,14,15,16,17,18]$. We abbreviate $\operatorname{det} g:=\operatorname{det}(g a b)$.

Proposition 3.1 Variation of $L|\operatorname{det} g|^{\frac{1}{2}}$ with respect to $g$ yields the Einstein equation in the form

$$
\kappa\left(R i c-\frac{1}{2} R g\right)=\phi^{*} h-e g .
$$

If $m=\operatorname{dim} X \geq 3$ then this is equivalent to

$$
\kappa R i c=\phi^{*} h .
$$

Variation of $L|\operatorname{det} g|^{\frac{1}{2}}$ with respect to $\phi$ yields

$$
\operatorname{tr}(\nabla d \phi)=0,
$$

where $\nabla d \phi$ is the second fundamental form of $\phi$ and the trace tr refers to the metric $g$.

The right-hand side of $(3.2)$

$$
T:=\phi^{*} h-e g
$$

is called energy-momentum tensor of $\phi$. There holds $e=\frac{1}{2} \operatorname{tr}\left(\phi^{*} h\right)$ and (3.5) is equivalent to

$$
T-(m-2)^{-1}(\operatorname{tr} T)=\phi^{*} h .
$$

Proposition 3.2 From the field equation (3.4) for $\phi$ there follows that $T$ is divergence-free:

$$
\nabla^{b} T_{a b}=0
$$

The proof follows from the identity

$$
\nabla^{b} T_{a b}=h_{i j}\left(\partial_{a} \phi^{i}\right)(\operatorname{tr} \nabla d \phi)^{j} .
$$




\section{Energy conditions for a wave map}

Let now the metric $g$ have Lorentzian signature $(+-\ldots-)$.

Definition 4.1 Let a symmetric two-form $T=T_{a b} d x^{a} d x^{b}$ on $X$ be interpreted as an energy-momentum tensor field and let $v=v^{a} \partial_{a}$ be a unit timelike vector field on $X$, i.e., $v_{a} v^{a} \equiv g_{a b} v^{a} v^{b}=1$. Then $T(v, v) \equiv$ $T_{a b} v^{a} v^{b}$ is called energy density, $I:=T(\cdot, v)$ with components $I_{a}:=T_{a b} v^{b}$ is called momentum one-form, and $J:=I-T(v, v) v$ with components $J_{a}:=I_{a}-T(v, v) v_{a}$ is called proper momentum one-form.

Physically, $v$ is interpreted as the unit tangent vector to the world line of an observer. This observer measures the quantities $T(v, v), I, J$.

Every unit timelike vector field $v=v^{a} \partial_{a}$ on $X$ gives rise to a positive definite metric $g^{+}=g_{a b}^{+} d x^{a} d x^{b}$ on $X$ with components $g_{a b}^{+}=2 v_{a} v_{b}-g_{a b}$. The inverse $\left(g_{+}^{a b}\right):=\left(g_{a b}^{+}\right)^{-1}$ has a representation $g_{+}^{a b}=2 v^{a} v^{b}-g^{a b}$.

Theorem 4.1 Consider the energy-momentum tensor

$$
T=\phi^{*} h-e g
$$

of a map $\phi: X \longrightarrow Y$ between $(X, g)$ and $(Y, h)$. The energy density equals

$$
T(v, v)=e_{+}:=e\left[\phi, g_{+}\right] \equiv \frac{1}{2} g_{+}^{a b}\left(\partial_{a} \phi^{i}\right)\left(\partial_{b} \phi^{j}\right) h_{i j} .
$$

It is a positive definite quadratic form in $d \phi$. The momentum one-form I obeys the estimate

$$
e^{2} \leq|I|^{2} \leq e_{+}^{2}
$$

where

$$
|I|^{2}:=I_{a} I^{a} \equiv g^{a b} I_{a} I_{b}
$$

Proof:

Let us abbreviate $f:=\phi^{*} h$ with components $f_{a b}:=\left(\phi^{*} h\right)_{a b}=\left(\partial_{a} \phi^{i}\right)\left(\partial_{b} \phi^{j}\right) h_{i j}$. We calculate

$$
\begin{array}{rlc}
T(v, v) & = & T_{a b} v^{a} v^{b}=\left(f_{a b}-e g_{a b}\right) v^{a} v^{b} \\
& = & f_{a b} v^{a} v^{b}-e=\frac{1}{2}\left(2 v^{a} v^{b}-g^{a b}\right) f_{a b}=\frac{1}{2} g_{+}^{a b} f_{a b}=e_{+} .
\end{array}
$$

The proper momentum one-form $J$ is orthogonal to $v$, that means $J_{a} v^{a}=0$. Considering that, we find that

$$
\begin{array}{rlc}
0 & \leq & g_{+}^{a b} J_{a} J_{b}=-g^{a b} J_{a} J_{b} \\
& = & -g^{a b} J_{a}\left(I_{b}-e_{+} v_{b}\right)=-g^{a b} J_{a} I_{b} \\
& = & -g^{a b}\left(I_{a}-e_{+} v_{a}\right) I_{b}=-I_{a} I^{a}+e_{+}^{2} .
\end{array}
$$


Thus, the right-hand side inequality of (4.3) is proved. In order to prove the left-hand side of (4.3), we start with the remark that the matrix $\left(f_{a b}\right)$ is positive semi-definite. Let us consider a fixed point $x_{0} \in X$ and use coordinates $x^{a}$ such that

$$
v^{a}=\delta_{0}^{a}, \quad g_{+}^{a b}=\delta^{a b}
$$

in that very point $x_{0}$. Actually, such coordinates exist. The following $2 \times 2$ subdeterminants of $\left(f_{a b}\right)$ are non-negative:

$$
\begin{gathered}
f_{00} f_{11}-f_{10} f_{10} \geq 0, \\
f_{00} f_{22}-f_{20} f_{20} \geq 0, \\
\cdots \\
f_{00} f_{m m}-f_{m 0} f_{m 0} \geq 0 .
\end{gathered}
$$

Let us sum up:

$$
f_{00} f_{a a}-f_{a 0} f_{a 0} \geq 0 .
$$

Here a summation convention applies to the index $a$ and the coordinate conditions (4.5) are assumed. The inequality (4.6) can be can be brought into a coordinate-invariant form

$$
g_{+}^{a b}\left(f_{a b} v^{c} v^{d} f_{c d}-v^{c} f_{a c} v^{d} f_{b d}\right) \geq 0 .
$$

Here we insert

$$
\begin{gathered}
g_{+}^{a b} f_{a b}=2 e_{+}, \quad v^{c} v^{d} f_{c d}=e+e_{+}, \\
v^{c} f_{a c}=v^{c}\left(T_{a c}+e g_{a c}\right)=I_{a}+e v_{a}, \\
g_{+}^{a b}\left(I_{a}+e v_{a}\right)\left(I_{b}+e v_{b}\right)=2 e_{+}\left(e+e_{+}\right)+e^{2}-|I|^{2} .
\end{gathered}
$$

Taking all this together, the left-hand side inequality of (4.3) follows.

The conditions

$$
T(v, v) \geq 0, \quad|I|^{2} \geq 0
$$

together form the dominant energy condition, which expresses that the energy density is non-negative and that the momentum $I$ is causal. The latter condition physically means that the momentum $I$ propagates with a velocity which is not greater than the velocity of light.

The so-called strongy energy condition also holds in the present situation, namely in the form

$$
(m-2) T(v, v) \geq \operatorname{tr} T
$$


Theorem 4.2 Consider the energy-momentum tensor $T=T_{a b} d x^{a} d x^{b}$ to $\phi$ as above and lightlike vector fields $l=l^{a} \partial_{a}, n=n^{a} \partial_{a}$ such that $l^{a} n_{a} \equiv$ $g_{a b} l^{a} n^{b}=1$. Then

$$
T(l, l) \equiv T_{a b} l^{a} l^{b}=h\left(\phi_{*} l, \phi_{*} l\right) \geq 0,
$$

and the one-form $I:=T(\cdot, l)$ with components $I_{a}:=T_{a b} l^{b}$ obeys

$$
0 \leq|I|^{2} \leq 2 T(l, l) T(l, n) .
$$

\section{Proof:}

Assertion (4.7) follows from

$$
T(l, l)=\left(f_{a b}-e g_{a b}\right) l^{a} l^{b}=f_{a b} l^{a} l^{b}=h_{i j}\left(l^{a} \partial_{a} \phi^{i}\right)\left(l^{b} \partial_{b} \phi^{j}\right) .
$$

The projection tensor $p$ with components

$$
p_{a b}:=l_{a} n_{b}+n_{a} l_{b}-g_{a b}
$$

is a useful tool. It is orthogonal to $l$ and $n$, i.e.

$$
p_{a b} l^{b}=p_{a b} n^{b}=0
$$

and is positive semidefinite. Hence

$$
0 \leq p^{a b} I_{a} I_{b}=\left(2 l^{a} n^{b}-g^{a b}\right) I_{a} I_{b}=2 T(l, l) T(l, n)-|I|^{2},
$$

which proves the right-hand side inequality of (4.8).

Below we will also use

$$
p^{a b} T_{a b}=\left(2 l^{a} n^{b}-g^{a b}\right) T_{a b}=2 T(l, n)-\operatorname{tr} T=2 T(l, n)+(m-2) e .
$$

Let us, in order to complete the proof, consider a fixed point $x_{0} \in X$ and use coordinates $x^{a}$ such that

$$
l^{a}=l_{0}^{a}, \quad n^{a}=\delta_{1}^{a}, \quad\left(p_{a b}\right)=\operatorname{diag}(0,0,1, \ldots, 1)
$$

in that very point $x_{0}$, where diag indicates a diagonal matrix. Formally the same $2 \times 2$ subdeterminants of $\left(f_{a b}\right)$ as in the preceding proof are nonnegative. Their sum is now in another way transformed into a coordinateinvariant form

$$
p^{a b}\left[f_{a b}\left(l^{c} l^{d} f_{c d}\right)-\left(l^{c} f_{a c}\right)\left(l^{d} f_{b d}\right)\right] \geq 0 .
$$


Here we insert

$$
\begin{gathered}
p^{a b} f_{a b}=p^{a b}\left(T_{a b}+e g_{a b}\right)=p^{a b} T_{a b}-(m-2) e=2 T(l, n), \\
l^{c} l^{d} f_{c d}=T(l, l), \quad p^{a b}\left(l^{c} f_{a c}\right)\left(l^{d} f_{b d}\right)=p^{a b} I_{a} I_{b}=2 T(l, l) T(l, n)-|I|^{2} .
\end{gathered}
$$

The result $|I|^{2}=g^{a b} I_{a} I_{b} \geq 0$ follows.

Physically, theorem (4.2) can be interpreted in terms of a fictional observer which moves faster and faster. In the limit, he reaches the velocity of light and $v$ turns to $l$. The energy density $T(l, l)$ then remains non-negative and the momentum $I$ remains causal.

Corollary 4.3 There holds $T(l, n) \geq 0$. Especially, $T(l, n)=0$ iff $I_{a}=$ $T(l, l) n_{a}$.

Proof:

The formulas (4.7), (4.8) imply $T(l, n) \geq 0$. If $T(l, n)=0$ then $|I|^{2}=0$ and

$$
0=p^{a b}\left(l^{c} f_{b c}\right)=p^{a b} I_{b}=\left(l^{a} n^{b}+n^{a} l^{b}-g^{a b}\right) I_{b}=T(l, l) n^{a}-I^{a}
$$

\section{Implications of the Einstein equation}

Let us study

$$
\kappa R i c=\phi^{*} h,
$$

for given background $(Y, h)$ as a relation between $g$ and $\phi$. Contraction with $g^{-1}$ yields

$$
\kappa R=2 e .
$$

Double contraction with a vector field $v=v^{a} \partial_{a}$ on $X$ yields

$$
\kappa \operatorname{Ric}(v, v)=h\left(\phi_{*} v, \phi_{*} v\right) .
$$

with the interpretation that $y=\phi(x)$ is to be inserted into the right-hand side of $(5.3) ; \phi_{*} v$ denotes the push-forward of $v$ with respect to $\phi$. As a conclusion, $\kappa R i c(v, v)$ is positive definite in $\phi_{*} v$ and is positive semi-definite in $v$.

Proposition 5.1 From (5.1) there follows that Ric and $d \phi$, interpreted as linear map, have the same rank:

$$
r:=\operatorname{rank}(\operatorname{Ric})=\operatorname{rank}(d \phi) .
$$


In particular:

$r=0$ iff $\phi$ is constant.

$r=m \equiv \operatorname{dim} X$ iff $\phi$ is an immersion.

$r=n \equiv \operatorname{dim} Y$ iff $\phi$ is a submersion.

$r=m=n$ iff $\phi$ is a local diffeomorphism.

The proof of (5.4) is an exercise in linear algebra.

Note that $r=0$ means that $(X, g)$ is Ricci flat, i.e., Ric $=0$.

Proposition 5.2 If (5.1) holds then the conditions

$$
\begin{gathered}
\operatorname{Ric}(v, v)=0, \\
\operatorname{Ric}(\cdot, v)=0, \\
\phi_{*} v=0,
\end{gathered}
$$

for a vector field $v=v^{a} \partial_{a}$ on $X$, are equivalent to each other. Moreover, they imply

$$
£_{v} R i c=0,
$$

where $£_{v}$ denotes the Lie derivative with respect to $v$.

\section{Proof:}

Equation (5.3) in components reads

$$
\kappa R_{a b} v^{a} v^{b}=h_{i j}\left(\phi_{*} v\right)^{i}\left(\phi_{*} v\right)^{j}
$$

where $\left(\phi_{*} v\right)^{i}=v^{a} \partial_{a} \phi^{i}$. Moreover, (5.1) implies

$$
\kappa R_{a b} v^{b}=h_{i j}\left(\partial_{a} \phi^{i}\right)\left(\phi_{*} v\right)^{j} .
$$

These formulas and the definiteness of $h$ give the first assertion. Next, we use comoving coordinates $x^{a}$, which are adapted to $v$, that means $v^{a}=\delta_{0}^{a}$, and we get

$$
\begin{gathered}
\left(\phi^{*} v\right)^{i}=v^{a} \partial_{a} \phi^{i}=\partial_{0} \phi^{i} \\
\kappa £_{v} R_{a b}=\kappa \partial_{0} R_{a b}=\partial_{0}\left(\partial_{a} \phi^{i} \partial_{b} \phi^{j} h_{i j}\left(\phi^{k}\right)\right) .
\end{gathered}
$$

If $\partial_{0} \phi^{i}=0$ then also $\partial_{0} R_{a b}=0$. This fact can be translated into the second assertion.

Proposition 5.3 If the Ricci tensor vanishes on the vectors of some integrable distribution in the tangent bundle $T X$ and (5.1) holds then $\phi$ is constant on each leaf of the foliation to the distribution. 


\section{Proof:}

A distribution of rank $s$ in $T X$ is integrable iff it admits adapted coordinates $x^{a}$, which means that the distribution is locally spanned by the coordinate vectors $\partial_{1}, \partial_{2}, \ldots, \partial_{s}$. The assumption becomes

$$
\operatorname{Ric}\left(\partial_{a}, \partial_{b}\right)=0 \quad \text { for } a, b=1,2, \ldots, s .
$$

Proposition (5.2) then implies

$$
\partial_{1} \phi^{i}=0, \partial_{2} \phi^{i}=0, \ldots, \partial_{s} \phi^{i}=0 .
$$

Hence $\phi^{i}$ does not depend on $x^{1}, x^{2}, \ldots, x^{s}$ and is constant if the point $x$ varies in a leaf of the foliation, i.e., if $x^{s+1}=$ const., ..., $x^{m}=$ const.

Proposition 5.4 The Einstein equation (5.1) implies

$$
\kappa\left(\nabla_{a} R_{b c}+\nabla_{b} R_{c a}-\nabla_{c} R_{a b}\right)=2 h_{i j}\left(\nabla_{a} \partial_{b} \phi^{i}\right)\left(\partial_{c} \phi^{j}\right) .
$$

Proof:

Covariant differentiation of (5.1) gives

$$
\kappa \nabla_{c} R_{a b}=h_{i j}\left[\left(\nabla_{c} \partial_{a} \phi^{i}\right)\left(\partial_{b} \phi^{j}\right)+\left(\partial_{a} \phi^{i}\right)\left(\nabla_{c} \partial_{b} \phi^{j}\right)\right] .
$$

Some rearrangement yields (5.9).

Proposition 5.5 The Einstein equation (5.1) implies

$$
h(\operatorname{tr}(\nabla d \phi), d \phi)=0 .
$$

This equation for a submersion $\phi$ implies the harmonic or wave map equation (3.4).

\section{Proof:}

The Einstein tensor $R i c-\frac{1}{2} R g$ is divergence-free. This fact and (5.9) imply $2 h(\operatorname{tr}(\nabla d \phi), d \phi)_{c} \equiv 2 h_{i j} \operatorname{tr}(\nabla d \phi)^{i}\left(\partial_{c} \phi^{j}\right)=\kappa g^{a b}\left(\nabla_{a} R_{b c}+\nabla_{b} R_{c a}-\nabla_{c} R_{a b}\right)=0$.

If $\phi$ is a submersion, then the matrix with elements $h(\cdot, d \phi)_{i c}=h_{i j} \partial_{c} \phi^{j}$ has maximal rank and therefore (5.10) implies (3.4).

Proposition 5.6 If the Ricci tensor is covariantly constant, i.e., $\nabla$ Ric $=0$, and the Einstein equation (5.1) holds for a submersion $\phi$, then $\phi$ is totally geodesic, that means

$$
\nabla d \phi=0
$$




\section{Proof:}

If $\nabla$ Ric $=0$ then (5.9) reduces to $h_{i j}\left(\nabla_{a} \partial_{b} \phi^{i}\right)\left(\partial_{c} \phi^{j}\right)=0$. If, additionally, $\phi$ is a submersion, then (5.11) follows by means of the rank argument already used in the preceding proof.

The next proposition needs some preparation. A diffeomorphism $f$ : $X \longrightarrow X$ is called a Ricci symmetry iff

$$
f^{*} \text { Ric }=\text { Ric. }
$$

A vector field $v=v^{a} \partial_{a}$ on $X$ is called an infinitesimal Ricci symmetry or a Ricci collineation iff

$$
£_{v} R i c=0 .
$$

It is well known that the flow $f_{t}$ for $t \in I$ of a Ricci collineation $v$ is a oneparameter family of Ricci symmetries, i.e. $f_{t}^{*}$ Ric $=$ Ric. Here $x=f_{t}\left(x_{0}\right)$ by definition represents the solution of the initial-value problem

$$
\frac{d x}{d t}=v(x),\left.\quad x\right|_{t=0}=x_{0},
$$

and $I$ denotes an open interval which contains 0 .

Proposition 5.7 Let $v=v^{a} \partial_{a}$ be a Ricci collineation of $(X, g)$. Then the Einstein equation (5.1) implies that $\phi: X \longrightarrow Y$ is invariant under the flow $f_{t}$ for $t \in I$ of $v$, that means

$$
\phi \circ f_{t}=\phi
$$

\section{Proof:}

Let us again use comoving coordinates such that $v^{a}=\delta_{0}^{a}$. In these coordinates, $f_{t}$ is expressed by a translation $x^{0} \mapsto x^{0}+t, x^{1} \mapsto x^{1}, \ldots$, $x^{m-1} \mapsto x^{m-1}$. We know already from the proof of Proposition $5.2 \partial_{0} \phi^{i}=0$, i.e. each $\phi^{i}$ is independent of $x^{0}$. Hence $\phi^{i}=\phi^{i}\left(x^{a}\right)$ does not change under $x^{0} \mapsto x^{0}+t$, which is just expressed by (5.14).

The following definition is useful.

Definition 5.1 A property of subsets of a manifold $X$ holds globally if it is valid for every $X$. It holds locally if every point $x_{0} \in X$ has a neighborhood $U$ such that the property is valid for $U$. 
Theorem 5.8 Let $\phi: X \longrightarrow Y$ be a submersion between smooth manifolds $X, Y$ and let $Y$ be equipped with a positive definite metric $h$.

1 - Locally there is a positive definite metric $g$ on $X$ such that $\phi$ becomes a harmonic map $(X, g)$ and $(Y, h)$.

2- Locally there is a Lorentzian metric $g$ on $X$ such that $\phi$ becomes a wave map.

\section{Proof:}

We consider (5.1) and use the fact that the problem of prescribed Ricci curvature is locally solvable in the two cases [19, 20]. More precisely: cf., eg.

1- Einstein's equation (5.1) locally has a positive definite solution $g$. It can be constructed, e.g., through some boundary value problem [19]. By assumption, $\phi$ is a submersion; Proposition 5.5 implies $\operatorname{tr}(\nabla d \phi)=0$.

2- Einstein's equation (5.1) locally has a Lorentzian solution $g$. It can be constructed, e.g., through some Cauchy initial value problem cf., e.g. [20]. An argument like in item 1 completes the proof.

\section{Product and warped product source manifolds}

Definition 6.1: The product $(X, g)$ of two (pseudo-) Riemannian manifolds $\left({ }^{\prime} X,{ }^{\prime} g\right),\left({ }^{\prime \prime} X,{ }^{\prime \prime} g\right)$ is given by $X={ }^{\prime} X \times{ }^{\prime \prime} X$ as a product of manifolds and by

$$
g(u, v)={ }^{\prime} g\left({ }^{\prime} u,{ }^{\prime} v\right)+{ }^{\prime \prime} g\left({ }^{\prime \prime} u,{ }^{\prime \prime} v\right)
$$

where $u, v$ are vector fields on $X,{ }^{\prime} u,{ }^{\prime} v$ are the push-forwards of $u, v$ with respect to the projection $X \longrightarrow{ }^{\prime} X$, and " $u,{ }^{\prime \prime} v$ are the push-forwards of $u, v$ with respect to the projection $X \longrightarrow{ }^{\prime \prime} X$

We write then

$$
\begin{gathered}
g={ }^{\prime} g \oplus " g, \\
\operatorname{dim}^{\prime} X={ }^{\prime} m, \quad \operatorname{dim}^{\prime \prime} X={ }^{\prime \prime} m, \quad m={ }^{\prime} m+{ }^{\prime \prime} m .
\end{gathered}
$$

We apply the index convention

$$
{ }^{\prime} a,{ }^{\prime} b^{\prime} c, \ldots=1,2, \ldots,{ }^{\prime} m ; \quad \text { " } a,{ }^{\prime \prime} b^{\prime \prime} c, \ldots={ }^{\prime} m+1,{ }^{\prime} m+2, \ldots, m .
$$

definition 6.2: The warped product $(X, g)$ is given by $X={ }^{\prime} X \times{ }^{\prime \prime} X$ like above and by

$$
g(u, v)={ }^{\prime} g\left({ }^{\prime} u,{ }^{\prime} v\right)+w^{2}{ }^{\prime \prime} g\left({ }^{\prime \prime} u,{ }^{\prime \prime} v\right),
$$


where the warping function $w$ is a map ${ }^{\prime} X \longrightarrow \Re$ with positive values $w>0$. We write then

$$
g={ }^{\prime} g \oplus w^{2}{ }^{\prime \prime} g
$$

The following is known.

Proposition 6.1 If $(X, g)$ is the product of $\left({ }^{\prime} X,{ }^{\prime} g\right),\left({ }^{\prime \prime} X,{ }^{\prime \prime} g\right)$ then the Einstein equation $\kappa R i c=\phi^{*} h \equiv h(d \phi, d \phi)$ decomposes into the two Einstein equations

$$
\kappa^{\prime} R i c=h\left({ }^{\prime} d \phi,{ }^{\prime} d \phi\right), \quad \kappa^{\prime \prime} \operatorname{Ric}=h\left({ }^{\prime \prime} d \phi,{ }^{\prime \prime} d \phi\right)
$$

and the orthogonality condition with respect to $h$

$$
h\left({ }^{\prime} d \phi,{ }^{\prime \prime} d \phi\right)=0
$$

in components

$$
\begin{aligned}
\kappa^{\prime} R_{a^{\prime} b}=h_{i j}\left(\partial_{a} \phi^{i}\right)\left(\partial_{{ }^{\prime}} \phi^{j}\right), \quad \kappa^{\prime \prime} R_{\prime^{\prime \prime} a^{\prime \prime} b} & =h_{i j}\left(\partial_{\prime^{\prime} a} \phi^{i}\right)\left(\partial_{\prime^{\prime} b} \phi^{j}\right), \\
h_{i j}\left(\partial_{\prime^{\prime}} \phi^{i}\right)\left(\partial_{\prime^{\prime} b} \phi^{j}\right) & =0 .
\end{aligned}
$$

Proposition 6.2 If $(X, g)$ is the warped product of $\left({ }^{\prime} X,{ }^{\prime} g\right),\left({ }^{\prime \prime} X,{ }^{\prime \prime} g\right)$ with warping function $w$ then the Einstein equation $\kappa$ Ric $=\phi^{*} h \equiv h(d \phi, d \phi)$ decomposes into

$$
\begin{gathered}
\kappa\left({ }^{\prime} R i c-{ }^{\prime \prime} m w^{-1 /} \nabla^{\prime} d w\right)=h\left({ }^{\prime} d \phi,{ }^{\prime} d \phi\right), \\
\kappa\left({ }^{\prime \prime} R i c-\frac{1}{" \prime m} w^{2-{ }^{\prime \prime} m{ }^{\prime \prime} \Delta w^{\prime \prime} m " \prime} g\right)=h\left({ }^{\prime} d \phi,{ }^{\prime \prime} d \phi\right), \\
\left.h{ }^{\prime} d \phi,{ }^{\prime \prime} d \phi\right)=0 .
\end{gathered}
$$

The trace parts of (6.5), (6.6) read

$$
\begin{gathered}
\kappa\left({ }^{\prime} R-{ }^{\prime \prime} m w^{-1 \prime} \Delta w\right)=2^{\prime} e, \\
\kappa\left({ }^{\prime \prime} R-w^{2-{ }^{\prime \prime} m \prime} \Delta w^{\prime \prime} m\right)=2^{\prime \prime} e .
\end{gathered}
$$

The proof is an exercise in higher differential geometry.

We say that $\phi:{ }^{\prime} X \times{ }^{\prime \prime} X \longrightarrow Y$ does not depend on ' $x$ iff the restriction $\phi\left(\cdot,{ }^{\prime \prime} x\right):{ }^{\prime} X \longrightarrow Y$ is a constant map for every ${ }^{\prime \prime} x \in{ }^{\prime \prime} X$. We say that $\phi$ does not depend on " $x$ with an analogous situation. 
Theorem 6.3 Let in the situation of proposition 6.2 the restriction $\phi\left({ }^{\prime} x, \cdot\right)$ : " $X \longrightarrow X$ be a submersion for every' $x \in{ }^{\prime} X$. Then $\phi$ does not depend on 'x.

\section{Proof:}

If every $\phi\left({ }^{\prime} x, \cdot\right)$ is a submersion then the quantities $h_{i j} \partial_{{ }^{\prime \prime} b} \phi^{j}$ in (6.4) form a matrix of maximal rank, and (6.4) implies $\partial^{\prime} a \phi^{i}=0$, which gives the assertion.

Theorem 6.4 Let in the situation of proposition 6.2 the first factor $\left({ }^{\prime} X,{ }^{\prime} g\right)$ be properly Riemannian and closed. Then the following holds.

(i) The symmetric two-form $\kappa^{\prime \prime}$ Ric is positive semi-definite.

(ii) If $\kappa^{\prime \prime}$ Ric is not everywhere positive then $w=$ const.

(iii) $\kappa \int_{{ }^{\prime} X} w^{\prime} R d^{\prime} v o l \geq 0$.

(iv) If $\int_{{ }^{\prime} X} w^{\prime} R d^{\prime} v o l=0$ then $w=$ const. and $\phi$ does not depend on ${ }^{\prime} x$.

\section{Proof:}

(i) Multiply (6.6) by $w^{\prime \prime} m-2$ and evaluate the two-forms at a vector field " $v \neq 0$ on " $X$ :

$$
\kappa\left(w^{\prime \prime} m-2 \text { " } \operatorname{Ric}\left({ }^{\prime \prime} v,{ }^{\prime \prime} v\right)-\left.\left.\frac{1}{{ }^{\prime \prime} m}\left({ }^{\prime} \Delta w^{\prime \prime} m\right)\right|^{\prime \prime} v\right|^{2}\right)=w^{\prime \prime} m-2 h\left({ }^{\prime \prime} d \phi\left({ }^{\prime \prime} v\right),{ }^{\prime \prime} d \phi\left({ }^{\prime \prime} v\right)\right) .
$$

Integrate this equation over ' $X$; the Laplacian term does not contribute, hence

$$
\kappa^{\prime \prime} \operatorname{Ric}\left({ }^{\prime \prime} v,{ }^{\prime \prime} v\right) \int_{{ }_{X}} w^{\prime \prime} m-2 d^{\prime} v o l=\int_{X_{X}} w^{\prime \prime} m-2 h\left({ }^{\prime \prime} d \phi\left({ }^{\prime \prime} v\right),{ }^{\prime \prime} d \phi\left({ }^{\prime \prime} v\right)\right) d^{\prime} v o l .
$$

Both the integrals are non-negative, hence $\kappa^{\prime \prime} \operatorname{Ric}\left({ }^{\prime \prime} v,{ }^{\prime \prime} v\right) \geq 0$ for every " $v$.

(ii) If "Ric(" $\left.v,{ }^{\prime \prime} v\right)\left({ }^{\prime \prime} x_{0}\right)=0$ for some point " $x_{0} \in{ }^{\prime \prime} X$ then

$$
\int_{{ }^{\prime} X} w^{\prime \prime} m-2 h\left({ }^{\prime \prime} d \phi\left({ }^{\prime \prime} v\left({ }^{\prime \prime} x_{0}\right)\right), " d \phi\left({ }^{\prime \prime} v\left({ }^{\prime \prime} x_{0}\right)\right) d^{\prime} v o l=0,\right.
$$

which implies " $d \phi\left({ }^{\prime \prime} v\left({ }^{\prime \prime} x_{0}\right)\right)=0$. Evaluation of (6.10) at the point " $x_{0}$ reduces $(6.10)$ to

$$
\left.\left.\left({ }^{\prime} \Delta w^{\prime \prime} m\right)\right|^{\prime \prime} v\left({ }^{\prime \prime} x_{0}\right)\right|^{2}=0 .
$$

We can assume $\left|{ }^{\prime \prime} v\left({ }^{\prime \prime} x_{0}\right)\right|^{2} \neq 0$; hence $w^{\prime \prime} m$ is a harmonic function. A harmonic function on a closed manifold is constant.

(iii) Multiply (6.8) by $w$ and integrate then over ' $X$. The Laplacian term does not contribute; hence

$$
\kappa \int_{{ }^{\prime} X} w^{\prime} R d^{\prime} v o l=\int_{{ }^{\prime} X} v^{\prime} e d^{\prime} v o l \geq 0
$$


(iv) The last integral vanishes only if ' $e=0$, which implies ' $d \phi=0$, hence $\phi\left(\cdot,{ }^{\prime \prime} x\right)=$ const for fixed " $x \in{ }^{\prime \prime} X$. But then ${ }^{\prime \prime} e\left(\cdot,{ }^{\prime \prime} x\right)=$ const and the standard separation argument can be applied to

$$
\kappa w^{2-{ }^{\prime} m \prime} \Delta w^{\prime \prime} m=\kappa^{\prime \prime} R-2^{\prime \prime} e .
$$

Thus both sides of (6.11) equal a constant c. Integration of

$$
\kappa^{\prime} \Delta w^{\prime \prime} m=c w^{\prime \prime} m-2
$$

yields $c=0$. Hence $w^{\prime \prime} m$ is a harmonic function on the closed manifold ' $X$. We find $w^{\prime \prime} m=$ const, $w=$ const.

Proposition 6.5 Let in the situation of the preceding theorem the second factor $\left({ }^{\prime \prime} X, " g\right)$ be properly Riemannian with vanishing scalar curvature: " $R=0$. Then $w=$ const and $\phi$ does not depend on " $x \in$ " $X$.

\section{Proof:}

Now equation (6.9) reduces to

$$
-\kappa^{\prime} \Delta w^{\prime \prime} m=2 w^{\prime \prime} m-2 " \prime e
$$

and we have ${ }^{\prime \prime} e \geq 0$. Integration over the closed manifold ' $X$ yields

$$
0=\int_{{ }_{X}} w^{\prime \prime} m-2 \prime \prime e d^{\prime} v o l
$$

which implies

$$
\text { "e } e={ }^{\prime \prime} \operatorname{tr} h(" d \phi, " d \phi)=0 .
$$

Hence " $d \phi=0$, i.e. $\phi$ does not depend on " $x$, and $w^{\prime \prime} m$ becomes a harmonic function. We arrive at $w^{\prime \prime} m=$ const, $w=$ const.

Example 6.1. Let in the situation of proposition 6.2 the first factor $\left({ }^{\prime} X,{ }^{\prime} g\right.$ ) be the unit circle $S^{1}$. It is a flat properly Riemannian closed manifold. Theorem 6.4 implies that $w=$ const and $\phi$ does not depend on ' $x$.

Example 6.2. A static metric $g=w^{2} d t^{2}-{ }^{\prime} g$ can be interpreted as a warped product metric on $X={ }^{\prime} X \times{ }^{\prime \prime} X$ where there the second factor " $X$ is one-dimensional. The proof of proposition 6.5 works, with a slight modification, for this case. Hence Einsteins equation $\kappa$ Ric $=\phi^{*} h$ implies that $w=$ const and $\phi$ does not depend on $t \in{ }^{\prime \prime} X$. 


\section{Source manifolds with radiation conditions}

Lichnerowicz [21] proposed conditions of pure radiation for a Lorentzian manifold $(X, g)$ :

$$
R_{a b c d} l^{d}=0, \quad R_{a b[c d} l_{e]}=0,
$$

where $R_{a b c d}$ are the components of the Riemann curvature tensor and $l=$ $l^{a} \partial_{a}$ is a lightlike vector field. Bel [22] proposed weaker conditions:

$$
R_{a b c d} l^{b} l^{d}=0, \quad l^{b} R_{a b[c d} l_{e]}=0, \quad l_{[f} R_{a b][c d} l_{e]}=0 .
$$

There are also radiation conditions for the Ricci tensor Ric or for the Einstein tensor $G:=R i c-\frac{1}{2} R g$ with components

$$
G_{a b}:=R_{a b}-\frac{1}{2} R g_{a b}
$$

namely

$$
\begin{array}{ll}
R_{a b} l^{b}=0, & R_{a[b} l_{c]}=0, \\
G_{a b} l^{b}=0, & G_{a[b} l_{c]}=0 .
\end{array}
$$

One of the present authors studied radiation conditions in [23, 24].

Theorem 7.1 If the Einstein equation $\kappa$ Ric $=\phi^{*} h$ holds then the conditions

$$
\begin{aligned}
& G_{a b} b^{b}=0, \\
& R_{a[b} l_{c]}=0
\end{aligned}
$$

for a lightlike vector field $l=l^{a} \partial_{a}$ are equivalent to each other.

\section{Proof:}

There is another lightlike vector field $n=n^{a} \partial_{a}$ such that $l^{a} n_{a}=1$. Then the sum $v:=l+n$ is timelike with $v^{a} v_{a}=2$ and $g_{a b}^{+}=v_{a} v_{b}-g_{a b}$ are the components of a positive definite metric $g_{+}$.

Let us consider

$$
\psi_{a}^{i}:=2 v^{b} l_{[a} \partial_{b]} \phi^{i} \equiv l_{a}\left(\phi_{*} v\right)^{i}-\partial_{a} \phi^{i}
$$

and calculate

$$
g_{+}^{a b} h_{i j} \psi_{a}^{i} \psi_{b}^{j}=-g^{a b} h_{i j} \psi_{a}^{i} \psi_{b}^{j}=h_{i j}\left(\phi_{*} v\right)^{i}\left(\phi_{*} l\right)^{j}-e=\kappa G_{a b} v^{a} l^{b} .
$$

Here we made use of

$$
g_{+}^{a b}=v^{a} v^{b}-g^{a b}, \quad v^{a} \psi_{a}^{i}=0
$$


and of $\kappa R i c=\phi_{*} h$. If $G_{a b} l^{b}=0$ then the above positive definite expression vanishes and we get

$$
\begin{gathered}
\psi_{a}^{i}=0, \quad \partial_{a} \phi^{i}=l_{a}\left(\phi_{*} v\right)^{i}, \\
\kappa R_{a b}=h_{i j}\left(\partial_{a} \phi^{i}\right)\left(\partial_{b} \phi^{j}\right)=l_{a} l_{b} h\left(\phi_{*} v, \phi_{*} v\right) .
\end{gathered}
$$

Conversely, from $R_{a b}=r l_{a} l_{b}$ with some scalar $r$ there follow $R=0$ and (7.1).

We here dwell again on the fact that to every smooth vector field $v=$ $v^{a} \partial_{a}$ on $X$ there are comoving coordinates $x^{a}=x^{0}, x^{1}, \ldots, x^{m-1}$, which means $v^{a}=\delta_{0}^{a}$. The adaptedness of coordinates is preserved by coordinate transformations of the form

$$
\bar{x}^{0}=x^{0}+f\left(x^{i}\right), \quad \bar{x}^{i}=\bar{x}^{i}\left(x^{j}\right) .
$$

We apply in this section the index convention

$$
\begin{gathered}
a, b, c, \ldots=0,1,2, \ldots, m-1, \\
i, j, k, \ldots=1,2, \ldots, m-1, \\
I, J, K, \ldots=2,3, \ldots, m-1 .
\end{gathered}
$$

Proposition 7.2 A Lorentzian manifold $(X, g)$ admits a lightlike ... hypersurface-orthogonal Killing vector field $l=l^{a} \partial_{a}$, that means

$$
l_{[c} \nabla_{a} l_{b]}=0, \quad \nabla_{(a} l_{b)}=0,
$$

iff in coordinates adapted to $l$ the metric assumes the form

$$
g=2 g_{01} d x^{0} d x^{1}+g_{i j} d x^{i} d x^{j},
$$

where $g_{01}, g_{i j}$ do not depend on $x^{0}$. The component $g_{01}=g_{01}\left(x^{k}\right)$ is invariant under gauge transformations (7.3) and the part $g_{I J} d x^{I} d x^{J}$ of (7.4) shows tensorial behavior under the part $\bar{x}^{i}=\bar{x}^{i}\left(x^{j}\right)$ of (7.3). Moreover, the matrix $\left(g_{I J}\right)=\left(g_{I J}\left(x^{k}\right)\right)$ is negative definite.

... covariantly constant vector field $l=l^{a} \partial_{a}$, that means $\nabla_{a} l_{b}=0$, iff in coordinates adapted to $l$ the metric assumes the form

$$
g=2 d x^{0} d x^{1}+g_{i j} d x^{i} d x^{j},
$$

where the components $g_{i j}$ do not depend on $x^{0}$.

... covariantly constant vector field $l=l^{a} \partial_{a}$ such that the Bel condition

$$
l_{[e} R_{a b][c d} l_{f]}=0
$$


holds iff there are coordinates adapted to $l$ such that

$$
g=2 g_{01} d x^{0} d x^{1}+g_{11}\left(d x^{1}\right)^{2}+2 g_{1 I} d x^{1} d x^{I}-d x^{I} d x^{I},
$$

where $g_{11}, g_{1 I}$ do not depend on $x^{0}$ and summation over $I$ is applied.

... covariantly constant vector field $l=l^{a} \partial_{a}$ such that the Lichnerowicz condition

$$
l_{[e} R_{a b] c d}=0
$$

holds iff there are coordinates adapted to $l$ such that

$$
g=2 g_{01} d x^{0} d x^{1}+g_{11}\left(d x^{1}\right)^{2}-d x^{I} d x^{I},
$$

where $g_{11}$ does not depend on $x^{0}$ and summation over $I$ is applied.

All these facts together with proof and additional information are given in the papers $[23,24]$.

A Lorentzian manifold $(X, g)$ which admits a covariantly constant lightlike vector $l=l^{a} \partial_{a}$ is called a plane-fronted gravitational wave with parallel rays, abbreviated $p p$-wave. Note that from $\nabla_{a} l_{b}=0$ and the Ricci identity there follows the Lichnerowicz condition

$$
R_{a b c d} l^{d}=0 .
$$

Theorem 7.3 A metric (7.4) satisfies an Einstein equation $\kappa$ Ric $=\phi^{*} h$ iff $g_{01}=g_{01}\left(x^{1}, x^{K}\right)$ is a harmonic function of $x^{K}=x^{2}, x^{3}, \ldots, x^{m-1}$ with respect to the positive definite metric (which depends on $x^{1}$ as a parameter) $-g_{I J} d x^{I} d x^{J}$.

\section{Proof:}

Some calculation gives the components

$$
R_{00}=0, \quad R_{01}=\frac{1}{2} \Delta g_{01}
$$

of the Ricci tensor Ric $=R_{a b} d x^{a} d x^{b}$, where $\Delta$ denotes the Laplace operator with respect to $-g_{I J} d x^{I} d x^{J}$. By proposition 5.2 , from $R_{00} \equiv R_{a b} l^{a} l^{b}=0$ and $\kappa$ Ric $=\phi^{*} h$ there follows $R_{01} \equiv R_{1 b} l^{b}=0$. The assertion follows.

Theorem 7.4 Let $m=\operatorname{dim} X=4$. A metric of the form (7.7) satisfies an Einstein equation $\kappa$ Ric $=\phi^{*} h$ iff it satisfies (7.8), that means iff it can be brought into the form (7.9). 


\section{Proof:}

Calculation of Ricci components gives $R_{I J}=0$; in particular $R_{I I}=0$ (without summation). By Proposition 5.2, from this and $\kappa R i c=\phi^{*} h$ there follows $R_{1 I}=0$. For $m=4$, there are only two independent curvature components of type $R_{i J K L}$, namely

$$
R_{1223}=-R_{13}, \quad R_{1323}=R_{12} .
$$

Thus we get $R_{i J K 2}=0$ which is expressed by (7.9) in a coordinate invariant way.

\section{Discussion}

The literature on harmonic or wave maps is very extensive. There are good surveys on harmonic maps $[4,5]$. Work on such maps in the role of matter fields coupled with gravitation began about 1980 [10,11]. One of the authors of this paper worked, with coauthors, already on this subject; we refer to the paper [17] and the unpublished preprint [25].

The Einstein equation $\kappa$ Ric $=\phi^{*} h$ or $\kappa G=T$, where $G=R i c-\frac{1}{2} R g$ denotes the Einstein tensor and $T=\phi^{*} h-e g$ the energy-momentum tensor of $\phi$ exhibits some remarkable properties:

- The rank of Ric, taken as a linear map, equals the rank of the differential $d \phi$ (Proposition 5.1).

- The symmetries of the Ricci tensor Ric and of the map $\phi$ are closely related to each other (Propositions 5.2, 5.3, 5.7).

- If $\phi$ is submersive then the Einstein equation implies the harmonic or wave map equation (Proposition 5.5; cf. also Proposition 5.6).

- In the Lorentzian case there are identities and estimates for the energy momentum tensor $T$ which indicate a physically good behavior of $T$ (Proposition 3.2, Theorems 4.1, 4.2).

- In the Lorentzian case there is a tendency to enhance radiation conditions. That means, the Einstein equation leads from one condition to a stronger condition (Section 7).

There is also a situation where the Einstein equation serves as an auxiliary construction: for a given submersion $\phi$ there locally exists a metric $g$ on $X$ which makes $\phi$ to a harmonic or wave map (Theorem 5.8).

One paper cannot touch all aspects of a subject. We did not discuss here:

- Bochner-Weitzenböck technique [5, 13, 14, 15],

- consequences of second variation formulas, 
- factorizations of the map $\phi[18]$,

- exact solutions $[7,8,9,10,11,12,13,14,15,16]$,

- coupling of $\phi$ to a gravitational theory other than Einsteins theory.

These topics are by far not exhausted and could be subjects of further research.

\section{Acknowledgments}

Former discussions and cooperations with T. Hirschmann and T. Deck are gratefully acknowledged. We thank the professors G. Huisken and A. D. Rendall for inspiring discussions.

\section{References}

[1] A. Macias, J. L. Cervantes-Cota and C. Lammerzahl, Exact Solutions and Scalar Fields in Gravity, Kluwer, Dordrecht (2001).

[2] V. Faraoni, Cosmology in Scalar-Tensor Gravity, Kluwer, Dordrecht (2004).

[3] A. D. Rendall, Partial Differential Equations in General Relativity, Oxford Univ. Press (2008).

[4] J. Eells and J. H. Sampson, Harmonic Mappings of Riemannian Manifolds, Amer. J. Math. 86 (1964), 109-160.

[5] J. Eells and L. Lemaire, Two Reports on Harmonic Maps, Worls Scientific, Singapore (1993).

[6] M. Struwe, Wave maps, Birhhauser, Basel (1997).

[7] P. Bizon, T. Chmaj and Z. Tabor, Dispersion and collapse of wave maps. Nonlinearity 13 (2000), 1411-1423.

[8] P. Bizon and A. Wasserman, Self-similar spherically symmertic wave maps coupled to gravity, Phys. Rev. D 62 (2000), 084031.

[9] P. Bizon and A. Wassermann, On the existence of self-similar spherically symmetric wave maps coupled to gravity, Class. Quant. Grav., 19 (2002), 3309-3321. 
[10] V. de Alfaro, S. Fubini and G. Furlan, Gauge theories and strong gravity, Il Nuovo Cim. A 50 (1979), 523-554.

[11] C. Omero and R. Percacci, Generalized non-linear $\sigma$-models in curved space and spontaneous compactification, Nucl. Phys. B 165 (1980), 351-364.

[12] M. Gell-Mann and B. Zwiebach, Spacetime compactification induced by scalars, Phy. Lett. B 141 (1984), 333-336.

[13] G. Ghika, Harmonic maps and submersions in local Euclidean gravity coupled to the $\sigma$-model, Rev. Roum. Phys., 31 (1986), 635-648.

[14] G. Ghikaand and A. Corciovei, Static solutions for the sigma-model coupled to gravity, Rev. Roum. Phys., 32 (1987), 827-835.

[15] G. Ghika and M. Visinescu, Four-dimensional $\sigma$-model coupled to the metric tensor field, Il Nuovo Cim. B 59 (1980), 59-73.

[16] S. Ianus and M. Visinescu, Spontaneous compactification induced by non-linear dynamics, Class. Quant. Grav., 3 (1986), 889-896.

[17] R. Schimming and T. Hirschmann, Harmonic maps from spacetimes and their coupling to gravitation, Astron. Nachr., 309 (1988), 311-321.

[18] A. P. Whitman, R. J. Knill and W. R. Stoeger, Some harmonic maps on pseudo-Riemannian manifolds, Internat. J. Theoret. Phys., 25 (1986), 1139-1153.

[19] D. DeTurck, Existance of metrics with prescribed Ricci curvature: local theory, Invent. Math., 65 (1981), 179-207.

[20] H. Friedrich and A. D. Rendall, The Cauchy Problem for the Einstein Equations, Springer, Berlin (2000).

[21] A. Lichnerowicz, Radiations en relativite generale, In Colloque de Royaumont (1959). Edition CNRS, Paris (1962).

[22] L. Bel, La radiation gravitationalle. In: Colloque de Royaumont (1959). Edition CNRS, Paris (1962).

[23] R. Schimming, Riemannsche Raume mit ebenfrontiger und mit ebener Symmetrie, Math. Nachr., 59 (1974), 129-162. 
[24] B. Fiedler and R. Schimming, Exact solutions of the Bach field equations of general relativity, Rep. Math. Phys., 17 (1980), 15-36.

[25] T. Deck and R. Schimming, Harmonic maps coupled to the Einstein equation, Preprint Universitat Mannheim (1991). 